\title{
Исследование адсорбции красителей материалами, полученными из отходов молотого кофе
}

\author{
Фам Тхань Минь ${ }^{1,2}$, Лебедева О.Е. ${ }^{1}$ \\ ${ }^{1}$ Белгородский государственный национальный исследовательский университет, Белгород \\ ${ }^{2}$ Институт ядерных исследований, Далат, Вьетнам
}

Поступила в редакцию 21.07.2017 г.

\begin{abstract}
Выполнена оценка сорбционной способности материалов, полученных из отходов кофе, по отношению к красителям метиленовому голубому и конго красному. Показано, что эффективность сорбции повышается после модифицирования отходов кофе путем обработки раствором пероксида водорода. Для исходных и модифицированных сорбентов изучено влияние рН на эффективность сорбции. Проанализированы изотермы адсорбции, показано, что они адекватно описываются моделью Ленгмюра. Установлено, что сорбция обоих красителей протекает в смешаннодиффузионном режиме и удовлетворительно описывается уравнением псевдовторого порядка.
\end{abstract} адсорбция.

Ключевые слова: отходы кофе, метиленовый голубой, конго красный, пероксид водорода,

\section{Investigation of dyes adsorption by materials obtained from spent coffee grounds}

\author{
Pham Thanh Minh ${ }^{1,2}$, Lebedeva O.E. ${ }^{1}$ \\ ${ }^{1}$ Belgorod State National Research University, Belgorod \\ ${ }^{2}$ Nuclear Research Institute, Dalat, Vietnam
}

The subject of the research is spent coffee grounds and their adsorption capacity. The main purpose of the research is modifying coffee wastes by hydrogen peroxide for obtaining sorbents of high efficiency, which are suitable for water purification from dyes. Properties of original coffee wastes and the samples obtained by a treatment with one molar hydrogen peroxide solution are compared in the article.

Adsorption capacity of the samples towards methylene blue and congo red was determined by static method. Dyes concentration in the solutions was determinened spectrophotometrically. The point of zero charge was determined by immersion technique using the procedure adapted to plant sorbents. Specific surface area of original and modified samples was determined by means of sorption of ethylene glycol monoethyl ether. For an estimation of functional groups of the sorbent surfaces IR-spectroscopy was applied.

The treatment with hydrogen peroxide was shown to lead to an increase of specific surface area of the sorbent. The nature of functional groups didn't change essentially after the treatment. Kinetic curves of adsorption were analyzed using conventional models of diffusion and chemical kinetics. Sorption of both dyes was found to occur in mixed diffusion regime and could be satisfactorily described by pseudo-second order equation. Adsorption isotherms of methylene blue and congo red were analyzed. Isotherms for both original and modified sorbents were demonstrated to fit Langmuir model. The results obtained can be applied in the processes of water purification. The conclusion was made that sorbents obtained from spent coffee grounds are prospective materials with high sorption capacity towards both to cationic and anionic dyes.

Keywords: spent coffee grounds, methylene blue, congo red, hydrogen peroxide, adsorption. 


\section{Введение}

В настоящее время проблемы рационального использования природных ресурсов становятся все более актуальными. В последние десятилетия появилась и развивается перспективная идея использования так называемых «сорбентов низкой стоимости», которые сами являются отходами каких-либо процессов. Применение для очистки воды сорбентов, полученных из отходов, позволяет снизить затраты и превращает отходы в полезное сырье.

Имеется значительное количество научно-исследовательских работ, посвященных разработке новых сорбентов из растительного сырья (шелухи и стеблей подсолнечника, кукурузных початков, рисовой шелухи и др.) [1-2]. Новым перспективным сырьем для приготовления сорбентов некоторые авторы считают отходы молотого кофе (кофейную гущу) [3-5]. Этот напиток широко распространен во всем мире, и вопросы утилизации кофейной гущи представляют практический интерес. В странах Юго-Восточной Азии, например, во Вьетнаме ресурсный потенциал этого сорбента довольно велик.

Отходы молотого кофе предлагают использовать в качестве сорбента без дополнительной обработки; так, авторы [3-5] показали, что отходы кофе обладают достаточно высокой эффективностью по отношению к сорбции красителей и катионов тяжелых металлов. В работе [6] отходы производства растворимого кофе использовали для адсорбции метиленового голубого.

Однако в ряде публикаций отходы кофе подвергают обезжириванию путем обработки раствором гидроксида натрия. Например, результаты [7] свидетельствуют, что при обработке отходов кофе щелочью увеличиваются пористость, площадь удельной поверхности и эффективность адсорбции красителя бриллиантового зеленого. В публикации [8] показано, что обработка гидроксидом натрия приводит к повышению сорбционной емкости по отношению к нитробензолу.

Другие способы модифицирования встречаются достаточно редко. Интересен опыт авторов [9], которые подвергали отходы кофе обработке пероксидом водорода; следует, однако, отметить, что задачей данного исследования было получение пищевых волокон, пригодных для диетического применения.

Целью настоящей работы было модифицирование отходов кофе пероксидом водорода для получения высокоэффективных сорбентов, пригодных для очистки сточных вод от красителей.

\section{Эксперимент}

Образцы отходов кофе были собраны после заваривания вьетнамского коммерческого молотого кофе сорта Робуста. Отходы кофе промывали дистиллированной водой, нагретой до $50^{\circ} \mathrm{C}$, для удаления водорастворимых загрязнителей. Промывку проводили до исчезновения окраски кофе и достижения нейтральной среды. Затем осадок отфильтровывали и сушили в конвекционной печи при температуре $105^{\circ} \mathrm{C}$ в течение 5 часов. Высушенные отходы кофе измельчали до порошкообразного состояния и просеивали через сито с отверстиями размером 0.315 мм. Далее эти образцы именуются ОКИ (отходы кофе исходные).

Для получения сорбентов, модифицированных обработкой раствором пероксида водорода, - (ОКП) - образцы ОКИ помещали в раствор $\mathrm{H}_{2} \mathrm{O}_{2}$ различной концентрации (0.05M-3M) на 24 часа. Затем промывали дистиллированной водой до практически нейтральной реакции. Сушили в конвекционной печи при температуре $105^{\circ} \mathrm{C}$ в течение 5 часов. 
Адсорбционную способность образцов по отношению к метиленовому голубому (МГ) и конго красному (КК) определяли статическим методом. Концентрацию красителей в растворах определяли спектрофотометрически с использованием прибора SPECORD 210Plus, Германия, регистрируя оптическую плотность при 666 нм (МГ) или 500 нм (КК). Величину рН растворов поддерживали на уровне 9 (МГ) и на уровне 4 (КК) добавлением $0.01 \mathrm{M} \mathrm{NaOH}$ или $0.01 \mathrm{M} \mathrm{HCl}$. Bce эксперименты выполняли при комнатной температуре $\left(22 \pm 2^{\circ} \mathrm{C}\right)$.

Точку нулевого заряда определяли иммерсионным методом по методике, адаптированной к растительным сорбентам [10]. Площадь удельной поверхности исходного и модифицированного образцов определяли методом сорбции моноэтилового эфира этиленгликоля [11]. ИК-спектры получены на инфракрасном спектрофотометре с преобразованием Фурье «IRPrestige-21» (Shimadzu, Япония).

\section{Обсуждение результатов}

В первоначальных экспериментах была выполнена оценка влияния концентрации пероксида водорода, использованного при модифицировании, на сорбционные свойства образцов по отношению к двум красителям - метиленовому голубому и конго красному. Полученные результаты приведены на рис. 1. Из рисунка видно, что повышение концентрации $\mathrm{H}_{2} \mathrm{O}_{2}$ свыше $1 \mathrm{M}$ нецелесообразно, так как снижает степень очистки воды от КК. В связи с этим в последующих экспериментах для модифицирования использовали $1 \mathrm{M}$ раствор пероксида водорода, результаты именно для этого образца приводятся далее без дополнительных пояснений.

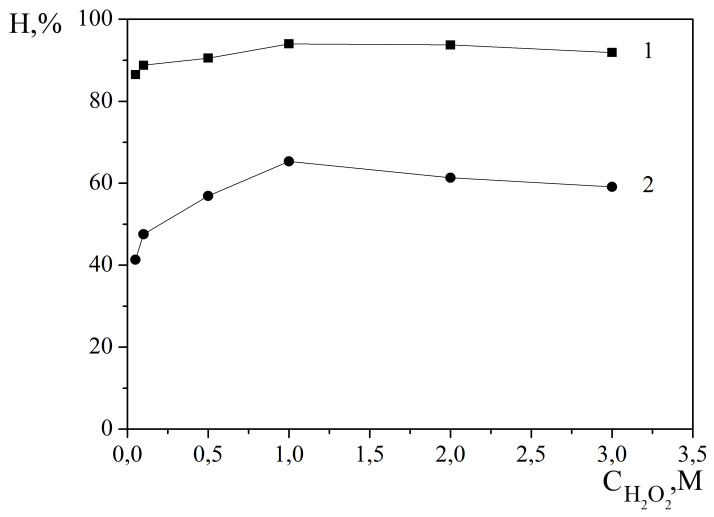

Рис. 1. Влияние концентрации модифицирующего $\mathrm{H}_{2} \mathrm{O}_{2}$ на эффективность адсорбции МГ (1) и КК (2) из растворов с исходной концентрацией 50 мг/дм².

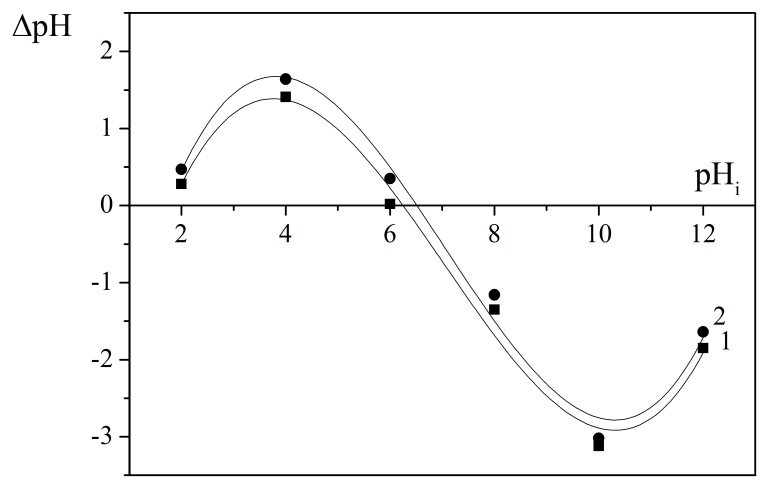

Рис. 2. Результаты определения точки нулевого заряда для образцов ОКИ (1) и ОКП (2).

Некоторые характеристики полученных сорбентов представлены в таблице 1 в виде сравнения исходного и модифицированного образцов. Можно отметить, что обработка пероксидом водорода привела к снижению минеральной составляющей.

Таблица 1. Физико-химические свойства сорбентов из отходов кофе

\begin{tabular}{|c|c|c|}
\hline Наименование & \multicolumn{2}{|c|}{ Значение } \\
\cline { 2 - 3 } & ОКИ & ОКП \\
\hline Зольность, \% мас. & 9.04 & 6.15 \\
\hline Влажность, \% мас. & 8.07 & 5.52 \\
\hline Дзета-потенциал при $\mathrm{pH}=7, \mathrm{mB}$ & -10.2 & -20.5 \\
\hline Площадь удельной поверхности, ${ }^{2} / \Gamma$ & 38 & 189 \\
\hline
\end{tabular}


Точки нулевого заряда для обоих образцов близки и составляют для ОКИ и ОКП 6.0 и 6.5 соответственно (рис. 2). При меньших значениях рН поверхность обоих образцов имеет положительный заряд, при рН выше значения точки нулевого заряда поверхность заряжена отрицательно. Поскольку метиленовый голубой является катионным красителем, а конго красный - анионным, естественно было ожидать, что адсорбция МГ будет происходить при высоких значениях рН, тогда как для КК оптимальное значение $\mathrm{pH}$ располагается в кислотной области.

Экспериментальные исследования подтвердили данное предположение. Оказалось, что эффективность сорбции обоих красителей высока в ожидаемом диапазоне $\mathrm{pH}$, причем внутри этого диапазона слабо зависит от величины $\mathrm{pH}$, особенно для модифицированного сорбента (рис. 3).
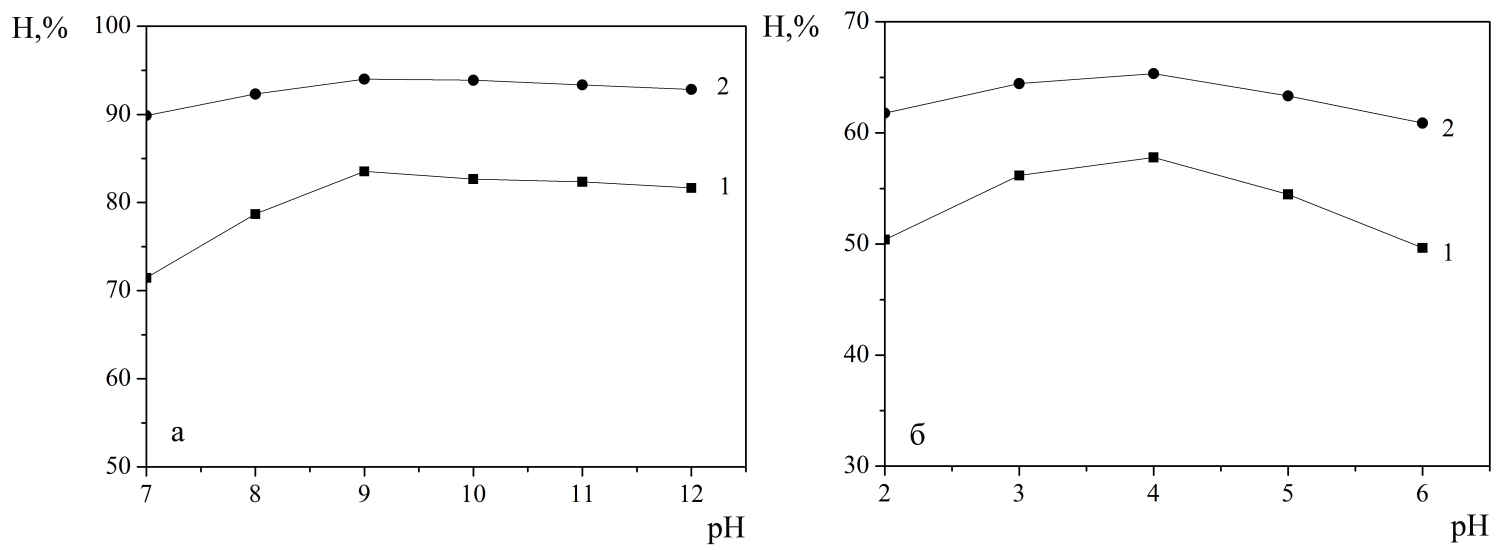

Рис. 3. Влияние рН на сорбционную эффективность ОКИ (1) и ОКП (2) по отношению к МГ (а) и КК (б). Исходная концентрация красителей 50 мг/дм ${ }^{3}$

Для оценки возможного механизма взаимодействия катионов МГ и анионов КК с функциональными группами отходов кофе были получены ИК-спектры сорбентов (рис. 4).

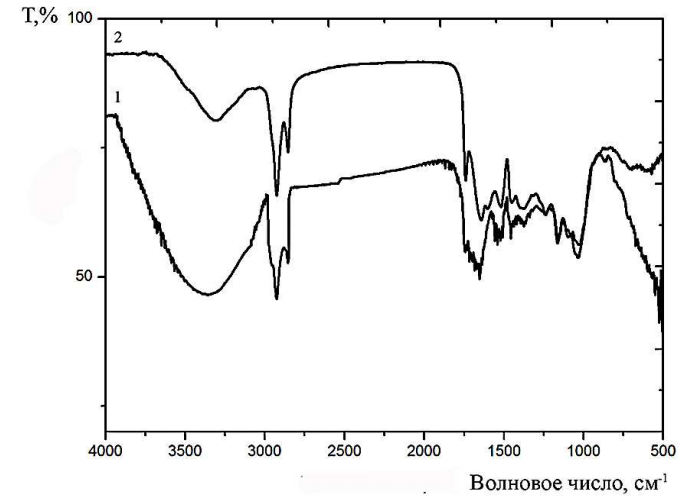

Рис.4. ИК-спектр ОКИ (1) и ОКП (2).

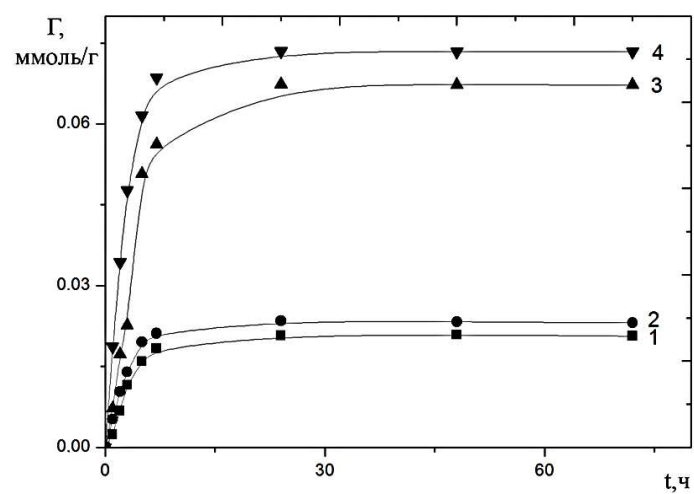

Рис. 5. Кинетические кривые адсорбции КК $(1,2)$ и МГ $(3,4)$ из растворов красителей с концентрацией 50 мг/дм ${ }^{3}$. Кривые 1 и 3 адсорбция на ОКИ, 2 и 4 - на ОКП.

Анализ спектров показал, что на поверхности материалов существует значительное количество карбонильных и карбоксильных групп (диапазон 1300-1750 $\mathrm{cm}^{-1}$ ), а также гидроксильные группы (широкие полосы при 3340-3360 $\mathrm{cm}^{-1}$ ). Эти группы могут быть потенциальными адсорбционными центрами. Спектры исходного и модифицированного образцов между собой различаются слабо; более 
высокая интенсивность полосы при $3360 \mathrm{~cm}^{-1}$ в спектре исходного образца, возможно, объясняется более высоким содержанием влаги в нем: известно, что в этой области проявляются также колебания гидроксильных групп молекул воды.

Обработка пероксидом водорода привела к существенному росту площади поверхности сорбентов (таблица 1). Возможно, это связано с изменением структуры и размеров пор. Представляло интерес оценить, повлияла ли обработка на характер диффузионных процессов в изучаемых образцах.

Кинетические кривые сорбции представлены на рис. 5. Можно видеть, что время достижения сорбционного равновесия составляет примерно 7 часов. Дальнейшее увеличение времени экспозиции до 3 суток не приводит к существенному изменению концентрации МГ и КК в растворах.

В рамках известных моделей диффузионной кинетики были построены зависимости $-\ln (1-F)=f(t)$ и $F=f\left(t^{1 / 2}\right)$, где $\mathrm{F}-$ степень завершения процесса, рассчитываемая по формуле $F=a_{t} / a_{e}$, в которой $a_{e} u a_{t}-$ количество адсорбата на единицу массы сорбента в состоянии равновесия и в момент времени $\mathrm{t}$ (рис. 6). Известно, что в случае преобладания внешнедиффузионного лимитирования процесса сорбции линейной является первая из приведенных зависимостей. Если лимитирующей стадией сорбции является внутренняя диффузия, то наблюдается линейная корреляция для второй зависимости [12]. В нашем случае удовлетворительные коэффициенты линейной корреляции получены для обеих зависимостей. Это позволяет предположить, что процесс протекает в смешаннодиффузионном режиме.

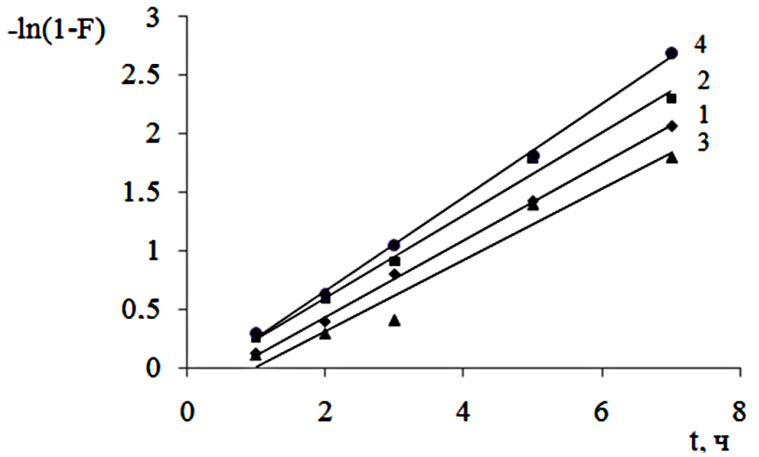

a

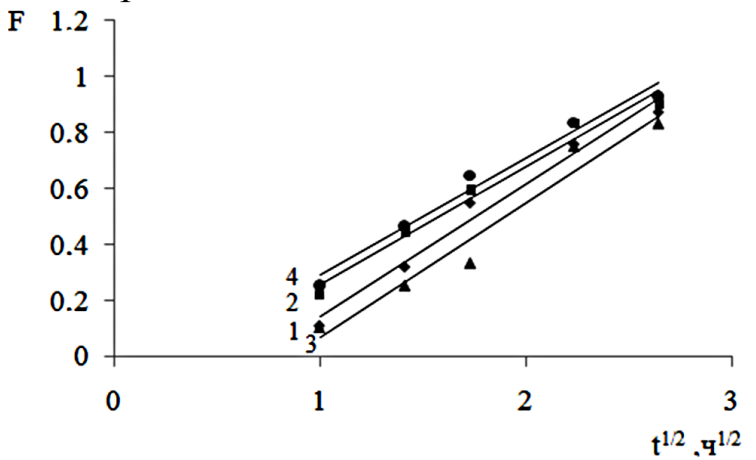

6

Рис. 6. Зависимость $-\ln (1-\mathrm{F})$ от времени $\mathrm{t}$ и $F$ от $t^{1 / 2}$ при сорбции КК $(1,2)$ и МГ $(3,4)$ на ОКП $(2,4)$ и ОКИ $(1,3)$

Для описания кинетики адсорбции на поверхности твердых веществ часто применяются модели псевдопервого и псевдовторого порядка. Полученные нами экспериментальные данные были графически обработаны в соответствующих координатах; основные результаты и коэффициенты линейной корреляции представлены в таблице 2. Очевидно, что модель кинетики псевдопервого порядка недостаточно хорошо описывает процесс адсорбции МГ и КК отходами кофе, о чем свидетельствуют сравнительно невысокие коэффициенты корреляции $\left(\mathrm{R}^{2}\right)$, полученные при обработке кинетических кривых в координатах $\ln \left(\Gamma_{\mathrm{e}}-\Gamma_{\mathrm{t}}\right)-\mathrm{t}$. Обработка кинетических кривых адсорбции в рамках модели кинетики псевдовторого порядка в координатах $\mathrm{t} / \Gamma_{\mathrm{t}}-\mathrm{t}$ дает значительно более высокие коэффициенты корреляции (таблица 2). Кроме того, сравнение экспериментальных величин $\Gamma_{\text {эксп }}$ со значениями $\Gamma_{\text {рас }}$, полученных при использовании моделей псевдопервого и псевдовторого порядков, свидетельствует о том, что во всех случаях модель псевдовторого порядка дает лучшее совпадение с экспериментальными результатами. Таким образом, кинетика адсорбции КК и МГ сорбентами ОКИ и ОКП адекватно описывается моделью кинетики псевдовто- 
рого порядка.

Таблица 2. Кинетические параметры адсорбции МГ и КК образцами из отходов кофе

\begin{tabular}{|c|c|c|c|c|c|c|c|}
\hline \multirow{2}{*}{$\begin{array}{l}\text { Образец- } \\
\text { адсорбат }\end{array}$} & \multirow{2}{*}{$\begin{array}{c}\Gamma_{\text {эксп, }} \\
\text { ммоль/Г }\end{array}$} & \multicolumn{3}{|c|}{$\begin{array}{c}\text { Кинетика псевдопервого } \\
\text { порядка }\end{array}$} & \multicolumn{3}{|c|}{$\begin{array}{c}\text { Кинетика псевдовторого } \\
\text { порядка }\end{array}$} \\
\hline & & $\mathrm{K}_{1}, \mathrm{\Psi}^{-1}$ & $\begin{array}{c}\Gamma_{\mathrm{pac},} \\
\text { мМОль/Г }\end{array}$ & $\mathrm{R}^{2}$ & $\begin{array}{c}\mathrm{K}_{2,} \\
\text { Гммоль }^{-1} \mathbf{u}^{-1}\end{array}$ & $\begin{array}{c}\Gamma_{\mathrm{pac}}, \\
\text { мМоль/Г }\end{array}$ & $\mathrm{R}^{2}$ \\
\hline ОКИ-КК & 0.021 & 0.046 & $10^{-5}$ & 0.539 & 14.1 & 0.022 & 0.992 \\
\hline ОКИ-МГ & 0.067 & 0.089 & $3 \cdot 10^{-4}$ & 0.788 & 2.7 & 0.073 & 0.990 \\
\hline ОКП-КК & 0.023 & 0.045 & $10^{-5}$ & 0.617 & 21.8 & 0.024 & 0.998 \\
\hline ОКП-МГ & 0.073 & 0.064 & $10^{-4}$ & 0.681 & 7.8 & 0.075 & 0.999 \\
\hline
\end{tabular}

С учетом данных о времени установления равновесия в изучаемых системах в дальнейших экспериментах сорбцию проводили на протяжении 24 часов. На рис. 7 представлены изотермы адсорбции МГ и КК на исходном ОКИ и модифицированном образце ОКП. Форма изотерм позволяет предположить, что адсорбция ограничивается монослоем. Видно, что предельная адсорбция на модифицированном образце выше, чем на исходном. Особенно наглядно это проявляется для адсорбции МГ. Этот факт является дополнительным подтверждением того, что воздействие пероксида водорода способствует формированию более развитой поверхности и пористости. Параметры изотерм, рассчитанные в рамках моделей Ленгмюра и Фрейндлиха, представлены в таблице 3.

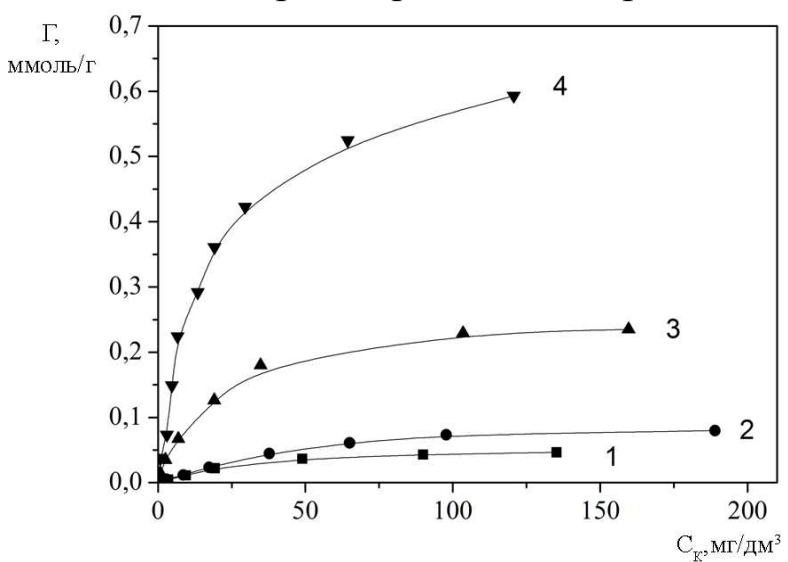

Рис.7. Изотермы адсорбции МГ и КК на образцах ОКИ-КК (1), ОКП-КК (2), ОКИ-МГ (3) и ОКП-МГ (4).

Таблица 3. Параметры изотерм адсорбции МГ и КК на образцах из отходов кофе, рассчитанные по моделям Ленгмюра и Фрейндлиха.

\begin{tabular}{|c|c|c|c|c|c|}
\hline \multirow{2}{*}{ Модель } & \multirow{2}{*}{ Параметры модели } & \multicolumn{2}{|c|}{ Метиленовый голубой } & \multicolumn{2}{|c|}{ Конго красный } \\
\cline { 2 - 6 } & & ОКИ & ОКП & ОКИ & ОКП \\
\hline \multirow{3}{*}{ Ленгмюра } & $\mathrm{K}_{\mathrm{L}}$ & 21.02 & 18.32 & 17.00 & 11.95 \\
\cline { 2 - 6 } & $\Gamma_{\text {мах, } \text { Ммоль/Г }} \mathrm{R}^{2}$ & 0.258 & 0.680 & 0.062 & 0.108 \\
\cline { 2 - 6 } Фрейндлиха & 0.996 & 0.993 & 0.990 & 0.983 \\
\cline { 2 - 6 } & $\mathrm{K}_{\mathrm{F}}$ & 4.59 & 16.41 & 1.37 & 1.96 \\
\cline { 2 - 6 } & $1 / \mathrm{n}$ & 0.632 & 0.586 & 0.705 & 0.700 \\
\hline & $\mathrm{R}^{2}$ & 0.916 & 0.915 & 0.959 & 0.965 \\
\hline
\end{tabular}

Из данных таблицы 3 видно, что адсорбция МГ и КК как на ОКИ, так и на ОКП хорошо описывается уравнением Ленгмюра. Вероятно, поверхность наших 
сорбентов можно считать достаточно однородной. Об этом же свидетельствует значение $1 / \mathrm{n}$ из уравнения Фрейндлиха.

\section{Заключение}

Таким образом, сорбенты, полученные из отходов молотого кофе, являются перспективными материалами с высокой сорбционной способностью как по отношению к катионным, так и к анионным красителям. Обработка пероксидом водорода существенно повышает площадь удельной поверхности образцов и их сорбционную способность, однако принципиально не влияет на природу функциональных групп поверхности сорбентов. Сорбция метиленового голубого и конго красного протекает в смешаннодиффузионном режиме и адекватно описывается кинетической моделью псевдовторого порядка. Адсорбция красителей в широком диапазоне концентраций ограничивается монослоем и удовлетворительно описывается моделью Ленгмюра.

\section{Список литературы}

1. Gupta V.K., Suhas // Journal of Environmental Management. 2009. Vol. 90. pp. 23132342.

2. De Gisi S., Lofrano G., Grassi M., Notarnicola M. // Sustainable Materials and Technologies. 2016. Vol. 9. pp. 10-40.

3. Kyzas G.Z. // Materials. 2012. Vol. 5. pp. 2069-2087.

4. Kyzas G.Z. // Materials. 2012. Vol. 5. pp. 1826-1840.

5. Kyzas G. Z., Lazaridis N. K., Mitropoulos A.Ch. // Journal of Engineering Science and Technology Review. 2012. Vol 5. pp. 66-75.

6. Franca A.S., Oliveira L.S., Ferreira M.E. // Desalination. 2009. Vol. 249. pp. 267-272.
7. Baek M.-H., Ijagbemi C.O., O S.-J., Kim D.-S. // Journal of Hazardous Materials. 2010. Vol. 176. pp. 820-828.

8. Dai Y., Zhang D., Zhang K. // Journal of the Taiwan Institute of Chemical Engineers. 2016. Vol. 68. pp. 232-238.

9. Vilela W.F., Leão D.P., Franca A.S., Oliveira L.S. // International Journal of Food Engineering. 2016. Vol. 2. pp. 42-47.

10.Fiol N., Villaescusa I. // Environ. Chem. Lett. 2009. Vol. 7. pp. 79-84.

11.Cerato A.B., Lutenegger A.J. // Geotechnical Testing Journal. 2002. Vol. 25. pp. 314320.

12.Алосманов Р.М. // Сорбичионые и хроматографические прочессы. 2010. Т. 10. № 3. C. $427-432$.

\section{References}

1. Gupta V.K., Suhas, Journal of Environmental Management, 2009, Vol. 90, pp. 23132342. DOI: $10.1016 /$ j.jenvman.2008.11.017

2. De Gisi S., Lofrano G., Grassi M., Notarnicola M., Sustainable Materials and Technologies, 2016, Vol. 9, pp. 10-40. DOI: https://doi.org/10.1016/j.susmat.2016.06.002

3. Kyzas G.Z., Materials, 2012, Vol. 5, pp. 2069-2087. DOI: 10.3390/ma5112069

4. Kyzas G.Z., Materials, 2012, Vol. 5, pp. 1826-1840. DOI: 10.3390/ma5101826

5. Kyzas G. Z., Lazaridis N. K., Mitropoulos A. Ch., Journal of Engineering Science and Technology Review, 2012, Vol 5(2), pp. 66-75.
6. Franca A.S., Oliveira L.S., Ferreira M.E., Desalination, 2009, Vol. 249, pp. 267-272. DOI: 10.1016/j.desal.2008.11.017

7. Baek M.-H., Ijagbemi C.O., O S.-J., Kim D.-S., Journal of Hazardous Materials, 2010, Vol. 176, pp. 820-828. DOI: 10.1016/j.jhazmat.2009.11.110

8. Dai Y., Zhang D., Zhang K., Journal of the Taiwan Institute of Chemical Engineers, 2016, Vol. 68, pp. 232-238. DOI: http://dx.doi.org/10.1016/j.jtice.2016.08.042

9. Vilela W.F., Leão D.P., Franca A.S., Oliveira L.S., International Journal of Food Engineering, 2016, Vol. 2, pp. 42-47. DOI: 10.18178/ijfe.2.1.42-47 
10.Fiol N., Villaescusa I., Environ. Chem. Lett., 2009, Vol. 7, pp. 79-84. DOI: 10.1007/s10311-008-0139-0

11.Cerato A.B., Lutenegger A.J., Geotechnical Testing Journal, 2002, Vol. 25, pp. 314-320. DOI: https://doi.org/10.1520/GTJ11087J

Лебедева Ольга Евгеньевна - профессор кафедры общей химии, д.х.н., профессор, Белгородский государственный национальный исследовательский университет, Белгород

Фам Тхань Минь - аспирант 2 года обучения, Белгородский государственный национальный исследовательский университет НИУ «БелГУ», Белгород. Институт ядерных исследований, Далат, Вьетнам.
12.Alosmanov R.M., Sorbtsionnye i khromatograficheskie protsessy, 2010, Vol. 10, No.3, pp. 427-432.

Lebedeva Olga Evgenievna - Professor, Doctor of chemical science, Belgorod State National Research University, Belgorod, OLebedeva@bsu.edu.ru

Pham Thanh Minh - PhD student of the $2^{\text {sd }}$ year, Belgorod State National Research University, Belgorod. Nuclear Research Institute, Vietnam. e-mail: 